\title{
Discussion
}

\section{Stahl Syndrome in Clinical Practice}

\author{
Samuel Stal, M.D., F.A.C.S. \\ Department of Plastic Surgery, Baylor College of Medicine/Texas Children's Hospital, Houston, TX 77030, USA
}

In the current medical climate, it has become very difficult to develop a large experience in the management of congenital anomalies, especially extremely rare ones such as Stahl syndrome. Irregardless, we all can learn something helpful using some of the tips and techniques described by Giuseppe Andrea Ferraro and colleagues to perfect our own otoplasty results.

Stahl syndrome is very apparent, with the irregular curvature secondary to the additional trifurcating crus $[1,2]$. As with other ear deformities, the thickness of the cartilage, the musculature of the ear, and the overlying skin relative to the surface area also contribute. What I find refreshing in this article is the "keeping it simple approach with a focused excision of aberrant cartilage ala Chongchet [1], redraping of skin, and use of the excised cartilage to splint the area.

Most important, I enjoyed the tried and true use of a postoperative customized splint to maintain the new skin and cartilage position. We all have seen the benefits of appropriate stenting, either to correct newborn lop ears or cleft nose (nasoalveolar molding) or postoperatively to maintain a curved septum or to help shape a skin graft [3]. All too often we minimize our effort with the postoperative dressing. For years, I have used elastic gauze (Spandage) for facial and skull dressings, and agree that patients love it because it is less constricting, easier to access, and more effective than classic mastoid dressings.

The surgical modifications described in the article differ from Chongchet's reconstruction by eliminating an anterior incision and using the resected cartilage to help reconstruct and maintain the dead space. Although the series was small, I enjoyed the focused simplified approach and the great use of customized splints and simplified dressings to maintain results and increase patient compliance. It is clear that to get a predictable result, appropriate diagnosis and focused treatment are essential. The authors should be complimented for their direct minimal approach to the correction of a significant problem.

\section{References}

1. Chongchet V: A method of antihelix reconstruction (no abstract). Br J Plast Surg 16:268-272, 1963

2. Furukawa M, Mitutani Z, Hamada T: A simple operative procedure for the treatment of Stahl's ear. $\mathrm{Br} J$ Plast Surg 38:544-545, 1985

3. Grayson BH, Maull D: Nasoalveolar molding for infants born with clefts of the lip, alveolus, and palate. Clin Plast Surg 31:149-158, 2004 